\title{
Construção de indicadores avaliativos de políticas de atenção à saúde da pessoa idosa vítima de acidentes e violência
}

\author{
Construction of indicators for assessing the policy \\ of reducing accidents and violence for the elderly care
}

Edinilsa Ramos deSouza ${ }^{1}$

Bruna Soares Chaves Correia ${ }^{1}$

${ }^{1}$ Centro Latino-Americano deEstudos de Violência e Saúde Jorge Careli, Escola Nacional deSaúde Pública Sergio Arouca, Fundação Oswaldo Cruz. Av. Brasil 4.036, sala 700,

M anguinhos. 21040-361 Rio deJaneiro RJ. edinilsa@claves.fiocruz.br
Abstract Thefollow article presents the methodology used to construct indicators to assess the implementation of the National Policy of M ortality Reduction by Accidents and Violence, of public health policies aimed at the elderly and the M ental Health Policy developed in the research entitled Diagnostic Analysis of Local H ealth Systems to M eet the Problems Caused by Accidents and Violence against Elderly. These indicators were applied in health services that meet elderly victims of accidents and violence in five Brazilian cities: Brasília, Curitiba, M anaus, Recife and Rio de Janeiro. It started with 124 indicatives to assistance level pre-hospital, hospital, rehabilitation and CAPS. Therewas a selection phase where indicatives without discriminatory capability wereeliminated. It wasalso decided by therelaxation of some criteria are creating new categories. After this step, a group of the experts validate the questionnaires created with these indicators by using Nominal Technical Group. We performed the Kruskal-Wallis test and a graphical analysis. In the final round, the indicators were grouped by similarity, building synthetic indices, 60 indicatives left. These methods can be used in other organizations to evaluate and adjust their health care based on public policies.

Key words Indicators, Violence, H ealth, Ederly, M ortality
Resumo 0 presenteartigo apresenta a metodologia de construção de indicadores avaliativos da implantação da Política Nacional de Redução da $M$ orbimortalidade por A cidentes eViolências, das políticas de saúde dirigidas ao idoso e de Saúde M ental foco da pesquisa Análise Diagnóstica de Sistemas Locais de Saúde para A tender aos A gravos Provocados por Acidentes e V iolências contra Idosos. Esses indicadores foram aplicados em serviços de saúde que atendem idosos víti mas de acidentes e violência em cinco capitais brasileiras: Brasília, Curitiba, M anaus, Recife e Rio de Janeiro. Inicialmente, foram criados 124 indicadores para os níveis de atendimento pré hospitalar, hospitalar, reabilitação e Centros de Atenção Psicossocial (CAPS). U m dos critérios de seleção foi eliminar os não discriminantes. Também foram flexi bilizadosal guns critérios criando-senovas categorizações. A pós essa etapa, elaboraram-sequestionários que foram repassadosa experts para validação por meio da Técnica Grupo Nominal. Realizou-se o teste de Kruskal-Wallis e uma análisegráfica. $N$ a rodada final, os indicadores foram aglutinados por semelhança construindo índices, restando sessenta indicadores. Essas ferramentas podem ser utilizadas em outras unidades para avaliar e adequar seu atendimento ao cumprimento dessas políticas públicas de saúde.

Palavras-chave Indicadores, Violência, Saúde, Idoso, M ortalidade 
Introdução

O conceito de acesso aos serviços de saúdeécomplexo e está relacionado à percepção das necessidades de saúde e da conversão dessas necessidades em demanda e destas em uso $0^{1,2}$. A queda da mortalidade, conjugada às melhorias nas condições de saúde provocadas por uma tecnologia médica mais avançada, bem como a universalização da seguridade social, maior acesso a serviços de saúde e outras mudanças tecnológicas, levou o idoso brasileiro a ter a sua expectativa de sobrevida aumentada ${ }^{3}$. Esse crescimento da população idosa, que vem ocorrendo desde as últimas décadas do século passado, tem pressionado o Sistema Ú nico de Saúde (SUS) a se adequar, exigindo maior complexidade tecnológica no atendimento a essa população. Em 1990, a população idosa correspondia a 7,2\% da população brasileira (10.384.803 idosos), passando para 9,6\% em 2007 (18.204.829 idosos) ${ }^{4}$.

Por se tratar de um fenômeno recente, a população idosa - que cada vez em maior número recorre aos serviços de saúde - tem enfrentado grande dificuldade para encontrar uma atenção de saúde que garanta as especificidades requeridas pelos complexos problemas característicos da sua condição, tanto pela falta ou insuficiência de serviços especializados como pela carência de profissionais devidamente capacitados para atendêla adequada e dignamente.

A magnitude do problema podeser demonstrada nos números registrados no Sistema de Internação Hospitalar (SIH) do M inistério da Saúde 5 . Em 2007, do total deinternações, 2.300.951 $(20,3 \%)$ foram de pessoas idosas, das quais $125.238(5,4 \%)$ foram por causas externas. As quedas representaram $53,7 \%$ das internações de idosos por causas externas, seguidas pelos acidentes detransporte, com 8,7\%. Em 2007, houve 68.484 internações por fratura no fêmur, das quais $47,7 \%$ foram de idosos.

Vale ressaltar que nesses dados constam apenas os problemas de saúde mais graves, que requereram internação em enfermaria de hospital. Além deles, existem os atendimentos nas emergências hospitalares e aqueles realizados pelos serviços móveis e ambulatoriais, que representam parcela ainda maior da atenção fornecida pela redeSUS.

No entanto, os serviços de saúde queatendem os idosos ainda permanecem com suas estruturas e organização pautadas em uma lógica "general ista" quenão contempla as necessidades eespecificidades $^{6}$ dessa clientela, as quais vêm sendo preco- nizadas em várias políticas e leis em que constam parâmetros e diretrizes nos quais o SUS deveria se pautar. As principais recomendações dessas políticas foram transformadas em indicadores.

Em termos gerais, os indicadores são medidas-síntese que contêm informação relevante sobre determinados atributos e dimensões. A construção de um indicador éum processo cuja complexidade pode variar desde a simples contagem direta de casos de determinada doença até o cálculo de proporções, razões, taxas ou índices mais sofisticados, como a esperança de vida ao nascer. É desejável que os indicadores possam ser analisados e interpretados com facilidade e que sejam compreensíveis pelos usuários da informação, especialmente gerentes, gestores e os que atuam no controle social do sistema de saúde.

Seforem gerados de forma regular e manejados em um sistema dinâmico, os indicadores de saúde constituem ferramenta fundamental para a gestão e a avaliação da situação de saúde, em todos os níveis. Constituem, assim, insumo para o estabel ecimento de políticas eprioridades mais bem ajustadas às necessidades de saúde da população ${ }^{7}$. Para a construção de bons indicadores, os dados que irão gerar essas informações devem ser completos efidedignos, além de haver a necessidade de profissionais qual ificados.

\section{Definição dos serviços}

Nos anos de 2003 a 2005, foi realizado um estudo inicial ${ }^{8} \mathrm{em}$ cinco capitais de cada uma das regiões do país (Brasília, Curitiba, Manaus, $R e-$ cifee Rio de aneiro), quevisou avaliar a implantação da Política Nacional de Redução deM orbimortalidade por Acidentes e Violências (PNRM AV). Nele, foram elaborados e validados indicadores avaliati vos da atenção às vítimas de violências em sistemas locais de saúde.

A referida pesquisa inspirou outro estudo ${ }^{6}$, realizado em 2007 e 2008, que focalizou especificamente $a$ aten ção à saúde prestada à população idosa em situação de violência, desta feita abordando inclusive o atendimento de saúde mental. Para isso foram considerados 174 serviços da rede SUS e conveniados que efetivamente ofereciam atendimento à pessoa idosa vítima de violência, sendo 81 do nível pré-hospitalar, 48 do hospitalar e 20 da reabilitação. Também fizeram parte da pesquisa 25 Centros de Atenção Psicossocial (CAPS) que oferecem atenção à saúde mental.

0 presente artigo visa, portanto, apresentar a metodologia de construção dos indicadores avaliativos criados, validados e aplicados nessa 
última investigação, envolvendo as mesmas cinco cidades da pesquisa inicial.

Processo de identificação dos indicadores existentes e análise dos parâmetros e diretrizes das políticas

Partiu-se de alguns indicadores da pesquisa de M inayo e Deslandes ${ }^{8}$ que foram classificados como imprescindíveis e que, de alguma forma, tinham relação com o atendimento ao idoso. Assim, foram resgatados 37 indicadores, sendo 12 do atendimento pré-hospitalar, 12 do hospitalar, nove do atendimento de reabilitação. Q uatro indicadores do nível pré-hospitalar usados nesse estudo também foram aproveitados para avaliar 0 atendimento dos CAPS.

0 passo seguinte foi a leitura aprofundada das políticas públicas de saúde particularmente dirigidas ao idoso:

. Política Nacional do Idoso $0^{9}$ - Lei no 8.842 , de 4 de janeiro de 1994 - Tem por objetivo asse gurar os direitos sociais do idoso, criando condições para promover sua autonomia, integração eparticipação efetiva na sociedade. Considera-se idoso, para os efeitos dessa lei, a pessoa maior de sessenta anos de idade.

- Política Nacional de Saúde da Pessoa Idosa $(\text { PNSI })^{10}$ - Portaria $\mathrm{n}$-2.528, de 19 de outubro de 2006 - Sua finalidade primordial é recuperar, manter epromover a autonomia ea independência dos indivíduos idosos, direcionando medidas coletivas e individuais de saúde para esse fim.

Ainda na área da saúde foram analisadas políticas específicas, como a Política Nacional de Redução da M orbimortalidade por Acidentes e Violências (PNRM AV) ${ }^{11}$, de 16 de maio de 2001, ea Política de Saúde M ental ${ }^{12}$, Lei no 36 , de 24 de julho de 1998, buscando identificar nelas as recomendações relativas ao idoso. Finalmente, também foi analisado o Estatuto do Idoso ${ }^{13}$, Lei $n$ 응 10.741, de $1^{\circ}$ de outubro de 2003, parâmetro maior da garantia dos direitos do idoso.

Com base na análise das diretrizes das próprias políticas em termos de estrutura, insumos, equipe, ações eaten dimento que deveria ser prestado, foram listados 87 indicadores avaliativos distribuídos da seguinteforma: 22 do atendimento pré-hospitalar, 13 do hospitalar, 17 do atendimento de reabilitação e 35 dos CAPS.

Somados aos indicadores recuperados da pesquisa anterior, foram elencados 34 indicadores avaliativos do atendimento pré-hospitalar; 25 do hospitalar; 26 da reabilitação; e ainda 39 indicadores para avaliar a atenção oferecida pelos CAPS, totalizando 124.
Processo inicial de aplicação e verificação do poder discriminante dos indicadores

Buscou-se verificar o desempenho dos indicadores criados realizando-se uma primeira aplicação aos dados de 101 questionários já respondidos pelos serviços de cada cidade ( 50 do nível préhospitalar, 19 do hospitalar, 11 de reabilitação e 21 dos (APS). Os indicadores que não se mostraram com capacidade discriminatória, isto é, aqueles para os quais todas as respostas obtidas enquadravam-se em apenas uma categoria, foram eliminados (oito do nível pré-hospitalar, um do hospitalar e quatro dos (APS).

Optou-se pela flexibilização dealguns indicadores, considerando-se que a atenção aos idosos vítimas de violências é um tema de recente reflexão no setor saúde e que alguns dos parâmetros estabelecidos nas políticas não estão ainda totalmente implantados. Desse modo, o critério para essa flexibilização foi o de que as unidades deveriam atender a pelo menos metade dos itens preconizados pelas políticas. Por exemplo: possuir seis dos 12 profissionais preconizados. Em seguida foi feita outra aplicação dos indicadores aos 174 serviços participantes ( 81 do nível pré-hospitalar, 48 do hospitalar, 20 de reabilitação e 25 dos CAPS), empregando-se, novamente, o critério de eliminação dos indicadores não discriminantes. N essa análise foram eliminados cinco indicadores do nível hospitalar, seis dos CAPS e sete dos níveis pré- hospitalar e reabilitação.

Os 86 indicadores que permaneceram (19 para cada um dos níveis do atendimento e 29 indicadores para os CAPS) foram incluídos em um questionário.

\section{Validação dos indicadores}

Esta etapa foi realizada por meio da Técnica Grupo Nominal ${ }^{14,15}$, que é uma metodologia qualitativa, participativa, de busca de consenso. Para isso, foram realizadas cinco oficinas presenciais, uma em cada cidadeincluída no estudo, nas quais foram envolvidos 47 especialistas ligados ao tema (média de 9,4 por oficina). Desse modo, foram consultados profissionais da equipe local da pesquisa, gestores e representantes dos distintos níveis do atendimento e de programas específicos do idoso das secretarias municipais de Saúde.

Inicialmente, foi solicitado a esses especialistas que avaliassem quantitativa equal itativamente cada um dos indicadores constantes em quatro listagens que Ihes foram fornecidas, relativas a cada um dos níveis do atendimento e uma para 
os serviços CAPS. Em termos qualitativos, eles avaliaram se 0 indicador era indispensável, necessário ou dispensável; de forma quantitativa, eles atribuíram uma nota de 0 a 6 correspondendo a: 0 = sem importância; 1 = quase sem impor tância; 2 = pouca importância; 3 = média importância; 4 = importante; 5 = muito importante; e6 = importantíssimo.

A pós essas atribuições de importânciae nota, os especialistas foram convidados a debaterem suas avaliações, argumentando os motivos dessa posição em relação àquele indicador. Ao término dessa discussão, cada um podia reavaliar sua opinião e modificá-la, registrando a nova avaliação no instrumento ou permanecer com a avaliação inicial.

A ideia é que, de acordo com sua expertise, esses especialistas focalizem a discussão nos pontos mais relevantes do indicador e que o consenso aumente entre eles.

\section{Análise dos indicadores}

Foi realizada uma análise preliminar das notasfinalmenteatribuídas aos indicadores pel osespecialistas usando-se o programa Excel $2003^{16}$, de modo a se buscar uma medida que expressasse a opinião conjunta dos especialistas e saber que níveis de consenso essas medidas obtiveram. Assim, usou-se a mediana ( $M \mathrm{~d}$ ), medida de posição central, como uma boa aproximação do grau de representatividade de cada um dos critérios, por dividir a série de tal forma que no mínimo $50 \%$ dos itens são iguais ou maiores do que ela; eo intervalo interquartílico (dq), medida dedispersão, como uma aproximação do grau de consenso ${ }^{17}$, expresso por $d q=q 3-q 1$, onde $q 1$ e $q 3$ representam 0 primeiro e o terceiro quartil, respectivamente.

Uma boa característica dessas medidas ( $M$ d e dq) é o fato de que elas não são influenciadas pela ocorrência de valores extremos. Foi considerada representativa das opiniões do grupo a $\mathrm{Md}$ entre $4 \mathrm{e} 6 . \mathrm{O}$ valor 3 foi adotado como intermediário, e valores baixos foram tidos como aqueles iguais ou inferiores $\mathrm{a} 2 . \mathrm{Um} \mathrm{dq} \leq 1$ sugere que 0 valor da mediana apresenta um alto grau de consenso, ao passo que um $\mathrm{dq} \geq 2$ indica que há uma concentração em torno de mais de uma posição, sem se aproximar de um consenso. De finiu-se como consenso aqueles valores que obtiveram um $d q \leq 1$. Assim, nenhum indicador foi eliminado, pois, segundo esses critérios, todos al cançaram o consenso pelos especialistas.

A fim deevidenciar diferenças estatisticamente significantes nas notas dos especialistas compa- rando-se os municípios, foi aplicado o teste não paramétrico deKruskal-Wallis, por meio do programa SPSS versão $15.0^{18}$, em que o nível de significância do p-valor foi igual a 5\%. Somentecinco indicadores pré hospitalares, quatro hospitalares, dois de reabilitação e três dos CAPS apresentaram diferença estatisticamente significante. A análise de correspondência ${ }^{19}$, método que permite a visualização gráfica das relações mais importantes de um conjunto de variáveis entre si, de maneira a estabelecer relações entre linhas e colunas da tabela de contingência, ajudou a evidenciar as relações esemel hanças existentes entre a relevância do indicador e os municípios, por meio de uma interpretação gráfica com o uso do programa $R$ versão $2.6^{20}$. Essa análise gráfica mostrou que, mesmo havendo diferença entreas notas dos municípios, elas não eram inferiores a 4 (importante). Os gráficos a seguir exemplificam a análise gráfica realizada. No Gráfico 1 , observa-se que os municípios de Recife e Manaus classificaram o indicador como importantíssimo, Rio de Janeiro como muito importante e Curitiba como importante.

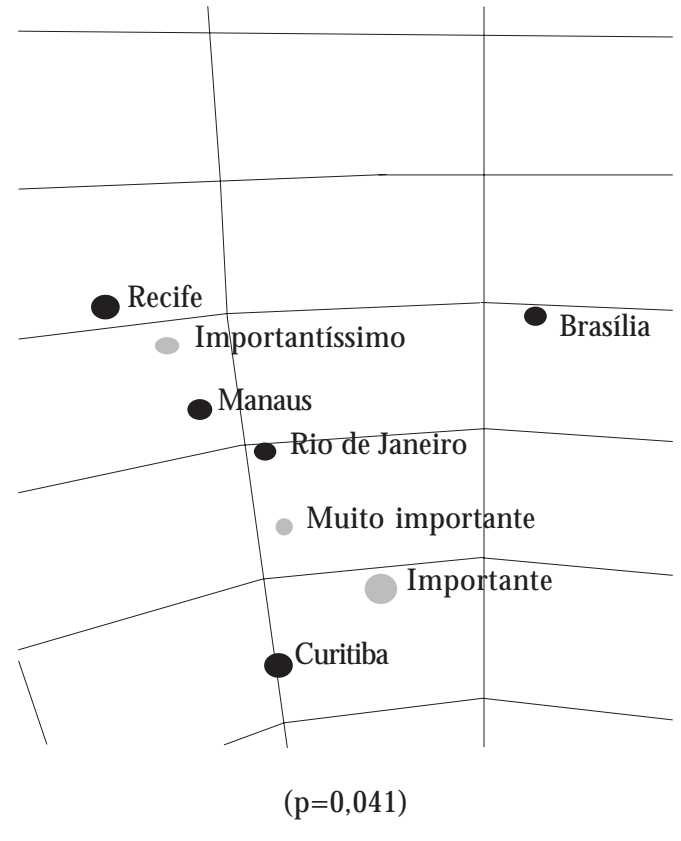

Gráfico 1. Análise das respostas dos especialistas sobre a importância do indicador "Proporção de unidades que realizam ações voltadas para o autocuidado" para 0 atendimento dos CAPS, segundo o município. 
No Gráfico 2, vê-seque os municípios deBrasília, Recife e $M$ anaus classificaram 0 indicador como importantíssimo, enquanto o município de Curitiba o classificou como muito importante.

No Gráfico 3, os municípios de Recife e M anaus classificaram o indicador como importantíssimo, enquanto o Rio de Janeiro o considera muito importante e Curitiba como importante.

Em seguida foi feita uma crítica relacionada à consistência das respostas, sendo invalidadas aquelas que não obtiveram concordância entre as duas opiniões dadas (ex.: nota 0 e indicador considerado indispensável). A avaliação qualitativa da importância foi categorizada em indispensável/necessário e dispensável, ea quantitativa ${ }^{3}$ em 4 e inferior a 4 . As novas variáveis categorizadas foram cruzadas e submetidas ao Teste de Associação Qui-quadrado de Pearson (com correção, pois as tabelas eram $2 \times 2$ ). Foram considerados significantes os valores de $p<0,05$. Nessa análise, nenhum indicador foi eliminado.

A fim de diminuir a quantidadeainda grande de indicadores, alguns deles foram aglutinados

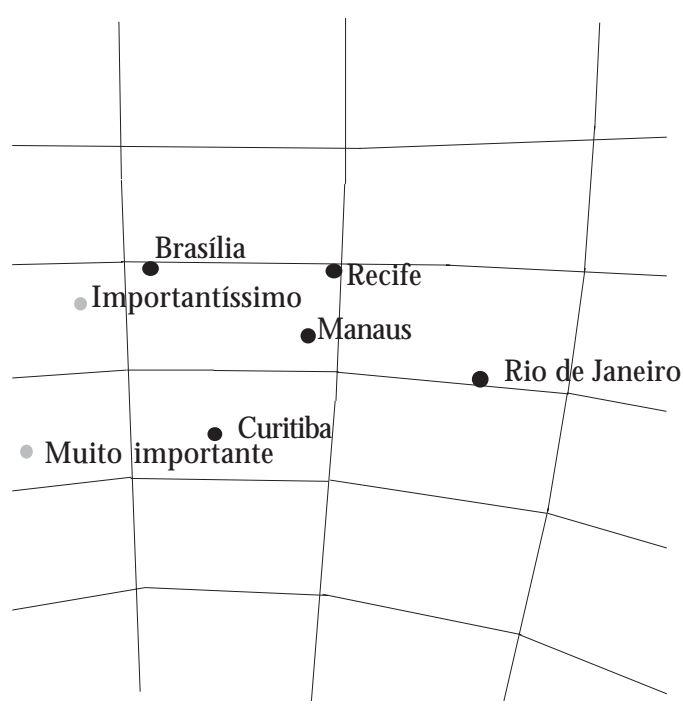

$(p=0,016)$

Gráfico 2. Análise das respostas dos especialistas sobre a importância do indicador "Proporção de unidades que realizam atividades de identificação e reconhecimento da rede de suporte social para a pessoa idosa" para o atendimento dos CAPS, segundo o município. por semelhança teórica. A construção desses índices deu-se da seguinte forma:

1. Foi atribuído um peso para cada indicador que comporia o índice. Esse peso foi calculado dividindo-se o número de variáveis que constituía cada indicador ( $\mathrm{N} v i$, onde: $\mathrm{i}=1 . . . \mathrm{n}$; $\mathrm{n}=$ número de indicadores) sobre o somatório do número de variáveis dos indicadores ( $\Sigma \mathrm{N}$ vi).

Ex.: Índicederecursos humanos para atendimento ao idoso é composto por dois indicadores $(i=1,2)$ :

Indicador 1 - Proporção de unidades que possuem equipe mínima de profissionais indispensáveis para seu funcionamento (médico, fisioterapeuta, assistente social, técnico/auxiliar de enfermagem) $-\mathrm{Nv}_{1}=4$

Indicador 2 - Proporção de unidades que possuem profissionais com formação em gerontologia/geriatria $-\mathrm{Nv}_{2}=1$

Peso do indicador $\mathrm{i}(\mathrm{Pi})=\mathrm{N}$ vi $/ \Sigma \mathrm{Nvi}$, onde: $i=1 . . . n ; n=$ número de indicadores

$$
P_{1}=\frac{4}{5}=0,8 \text { e } P_{2}=\frac{1}{5}=0,2
$$

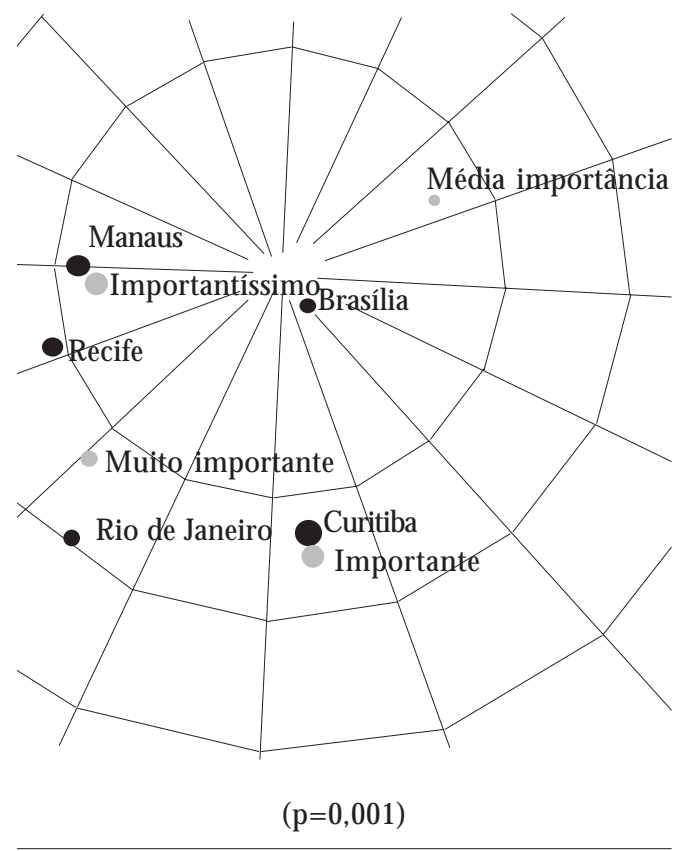

Gráfico 3. Análise das respostas dos especialistas sobre a importância do indicador "Proporção de unidades que encaminham pacientes para 0 Programa de Volta para Casa" para o atendimento dos CAPS, segundo o município. 
2. 0 índice decada unidade desaú de foi obtido da seguinte forma: multiplicou-se a quantidade devariáveis que cada unidadede saúde possuía de cada indicador ( $\mathrm{N}$ pi) por seu peso calculado ( $\mathrm{Pi}$ ) e dividiu-sepelo total de variáveis deste indicador ( $\mathrm{N}$ vi). Em seguida, somaram-se os resultados obtidos. 0 resultado dessa soma foi um escore entrezero e um.

Ex.: Uma unidade de reabilitação respondeu que possui três dos quatro profissionais da equipemínima indispensável (indicador 1 ) equepossui profissionais com formação em gerontologia/geriatria (indicador 2). Assim:

Índice de recursos humanos da unidade

$=\frac{(1 \times 0,2)}{1}+\frac{(3 \times 0,8)}{4}=0,2+0,6=0,8$

3. Para obter o escore do município, foi calculada a média dos escores de suas unidades.

N essa etapa final, os indicadores isolados passaram a compor os índices.

Dos 124 indicadores do rol inicial, restaram 60 (40 isolados e 20 sintéticos). Para os serviços do nível préhospitalar restaram 29 indicadores, sendo 13 para os serviços clínicos (noveindicadores equatro índices) e 16 para os Centros deAtenção Psicossocial (CAPS) da atenção em saúde mental, dos quais seis constituem índices. No ní- vel hospitalar atendimento clínico, permaneceram 12 indicadores, sendo noveisolados etrêsíndices. Os hospitais psiquiátricos ficaram com sete indicadores avaliativos do seu atendimento, dos quais dois são índices. No Nível da Reabilitação restaram 12 indicadores, sendo quatro deles índices. Ver listagem dos indicadores no apêndice.

\section{Consideraçõesfinais}

Essas ferramentas podem ser utilizadas pelos gestores para avaliar e adequar 0 atendimento de suas unidades ao cumprimento das políticas públicas de saúde particularmente dirigidas ao idoso, da Política Nacional de Redução da Morbimortalidade por Acidentes eViolências (PNRM AV), da Política de Saúde M ental e do Estatuto do Idoso. Também há a possibilidade da reprodução de sua aplicação em outras unidades.

Outro estudo importante seria a hierarquização dos indicadores de acordo com o seu grau de importância, o que nos forneceria informação a respeito do cumprimento das políticas elencadas como mais importantes.

Também seria interessante verificar até que ponto seria viável a aplicação desses indicadores no cotidiano dos serviços.

\section{Colaboradores}

ER Souza trabalhou na concepção e na redação do artigo; BSC Correia trabalhou na redação e na análise estatística do artigo. 


\section{Referências}

1. Ojanuga DN, Gilbert C. Women's access to health care in developing countries. Apud Pinheiro RS, Viacava $F$, Travassos $C$, Brito AS. Gênero, morbidade, acesso e utilização de serviços de saúde no Brasil. Cien Saude Colet 2002; 7(4):687-707. [periódico na Internet] [acessado $2010 \mathrm{abr}$ 29]. Disponível em: http://www.scielo.br/scielo.php?script=sci_arttext\& pid=S1413-81232002000400007\&lng=pt

2. Puentes-Markides $C$. Women and access to health care. Apud Pinheiro RS, Viacava F, Travassos C, Brito AS. Gênero, morbidade, acesso e utilização de serviços de saúde no Brasil. Cien Saude Colet 2002; 7(4): 687-707. [periódico na Internet] [acessado 2010 abr 29]. Disponível em: http://www.scielo.br/ scielo.php?script $=$ sci_arttext $\&$ pid $=$ S1413-8123200 2000400007\&lng=pt

3. Camarano AA. Envelhecimento da população brasileira: uma contribuição demográfica. In: Camarano AA. Sumário dos Resultados. Rio de Janeiro: M POG, I pea; 2002. [Texto para discussão, n. 858]. p. 22-23.

4. Departamento de Informática do SUS (Datasus). População Residente - Brasil. [site na Internet] [acessado 2009 nov 17]. Disponível em: http:// tabnet.datasus.gov.br/cgi/deftohtm.exe?ibge/cnv/ popbr.def

5. Departamento de Informática do SUS (Datasus) Morbidade hospitalar do SUS: por local de residência - Brasil. [site da Internet] [acessado 2009 nov 16]. Disponível em: http://tabnet.datasus.gov. $\mathrm{br} / \mathrm{cgi} /$ deftohtm.exe?sih/cnv/mrbr.def

6. Souza ER, coordenador. Análise diagnóstica de sistemas locais de saúde para atender aos agravos provocados por acidentes e violências contra idosos [ relatório de pesquisa]. Rio de Janeiro: Fiocruz/Claves; 2008.

7. Organização Pan-Americana da Saúde (Opas). Indicadores Básicos de Saúde no Brasil: conceitos e aplicações. Brasil: Rede Interagencial de Informações para a Saúde; 2002.

8. M inayo M CS, Deslandes SF, organizadores. Análise diagnóstica da política nacional de redução da morbimortalidade por acidentes e violências. Rio de Janeiro: Editora Fiocruz; 2007.

9. Brasil. Lei $n=8.842$, de 4 de janeiro de 1994. Dispõe sobre a Política Nacional do Idoso, cria o ConseIho Nacional do Idoso e dá outras providências. Diário Oficial da União 1994; 5 jan.
10. Brasil. Portaria no 2.528, de 19 de outubro de 2006. Política Nacional de Saúde da Pessoa Idosa. Diário Oficial da União 2006; 19 out.

11. Brasil. Portaria M S/GM № 737, de 16 de maio de 2001. Política Nacional de Redução da Morbimortalidade por Acidentes e Violências. Diário Oficial da União, Brasília 2001; 18 mai.

12. Brasil. Política de Saúde M ental. Lei $n=36$, de 24 de julho de 1998. Diário Oficial da U nião 1998; 24 jul.

13. Brasil. Estatuto do Idoso. Lei no 10.741 , de 10 de outubro de 2003. Diário O ficial da União 2003; 3 out.

14. Varela J. Los métodos de consenso en el sector sanitário. Gaceta Sanitária 1991; 5:114-116.

15. Jones J, Hunter S. Consensus methods for medical and health services research. British Med Journal 1995; 331:153-168.

16. Excel 2003. M icrosoft Office Excel 2003. [Software]. 1 CD-ROM .

17. Delgado P. Instituir a desinstitucionalização: o papel das residências terapêuticas na Reforma brasileira. In: Delgado P. Desinstitucionalização: a experiência dos serviços residenciais terapêuticos. Rio de Janeiro: IPUB, Universidade Federal do Rio de Janeiro; 2006. [Cadernos IPUB, n.22].

18. SPSS INC. Statistical Package Social Sciences 15.0. Chicago: SPSS Inc; 2006. [software]. 1 CD-ROM.

19. Carvalho MS, Strunchiner CJ. Análise de correspondência: uma aplicação do método à avaliação de serviços de vacinação. Cad Saude Publica 1992; $8(3): 287-301$

20. $R$ versão 2.6. $R$ Foundation for Statistical Computing 2007. [software] [site da Internet] [acessado 2009 mai 25]. Disponível em: www.r-project.org

Artigo apresentado em 02/02/2010

Aprovado em 22/05/2010

Versão final apresentada em 09/06/2010 
Apêndice

Indicadores do nível pré-hospitalar móvel e fixo:

. Proporção de unidades que possuem cadeira de rodas, acesso diferenciado para idosos e/ou portadores de necessidades especiais;

. Proporção de unidades que possuem integração com equipes do Programa de Saúde da Família (PSF) visando à manutenção da capacidade funcional da pessoa idosa;

. Proporção de unidades que oferecem ao idoso uma abordagem preventiva com aplicação de instrumentos de avaliação e testes de triagem para detecção dedistúrbios cognitivos, visuais, mobilidade, audição, depressão, comprometimento da funcionalidade, distúrbios nutricionais, isolamento social;

Proporção de unidades que atendem casos de idosos com transtornos mentais vítimas de acidentes e/ou violência e os referenciam;

. Proporção de unidades, exceto móveis, que oferecem de forma adequada e suficiente suporte e/ou acompanhamento aos idosos vítimas de acidentes e violências, às famílias/cuidadores deidosos vítimas de acidentes e violências, aos autores de violências contra idosos e às equipes que realizam o atendimento;

. Proporção de unidades, exceto móveis, que realizam busca ativa para casos mais graves de idosos em situação de violência quando há problemas de adesão ao tratamento;

. Proporção de unidades, exceto PS/Atendimento $24 \mathrm{~h}$, que realizam atendimento grupal, visitas domiciliares, atendimento à família, atividades comunitárias enfocando a integração do paciente na comunidade e sua inserção familiar e social;

Proporção de unidades, exceto móveis, que realizam atendimento clínico, cirúrgico, de urgência, traumato-ortopédico, atendimento geriátrico e gerontológico;

- Proporção de unidades cujos gestores/técnicos já ouviram falar e já leram a PNRM AV, a Política Nacional de Saúde da Pessoa I dosa e o Estatuto do Idoso;

. Índice de ações de prevenção de acidentes e violências - proporção de unidades que realizam ações de prevenção de violências e ações voltadas para prevenção de acidentes no domicílio e nas vias públicas;

. Índice de registro e análise dos dados - proporção de unidades que realizam o registro do atendimento aos acidentes/violências classificados de acordo com CID-10 ou DSM -IV e fazem análise sistemática deles;

. Índice de recursos humanos de serviço pré-hospitalar clínico específicos para atendimento ao idoso - proporção de unidades de serviço clínico que possuem recursos humanos disponíveis para o atendimento ao idoso eque possuem profissional com formação em gerontologia;

. Índice de identificação e notificação de casos de violência contra idosos - proporção de unidades que realizam ações voltadas para a identificação de casos de idosos vítimas de acidentes/violência; e que enviam a notificação de casos de violência contra idosos para autoridade policial ou M inistério Público ou Conselho M unicipal ou Estadual do Idoso ou Secretaria M unicipal ou Estadual de Saúde ou Secretaria de Desenvolvimento Social. CAPS:

Indicadores do atendimento pré-hospitalar dos

- Proporção de unidades que possuem cadeiras de rodas, acesso diferenciado para idosos e/ou portadores de necessidades especiais;

. Proporção de unidades que possuem profissionais com formação em gerontologia;

- Proporção de unidades do serviço CAPS II que realizam atividades de atendimento em oficinas terapêuticas, atividades comunitárias enfocando a integração do paciente na comunidade e sua inserção familiar e social;

- Proporção de unidades do serviço CAPS ad II que realizam atividades de atendimento grupal (grupo operativo, terapêutico, atividades socioterápicas, grupos de orientação, atividades de sala de espera, atividades educativas em saúde);

. Proporção de unidades que realizam atividades de atendimento de urgência;

. Proporção de unidades que realizam atividades de atendimento geriátrico e gerontológico;

. Proporção de unidades que realizam visitas domiciliares;

- Proporção de unidades que realizam busca ativa para casos mais graves de idosos em situação de violência quando há problemas de adesão ao tratamento;

. Proporção de unidades que encaminham pacientes para o Programa de Volta para Casa;

- Proporção de unidades cujos profissionais recebem capacitação para identificação e atendimento dos casos de idosos vítimas de acidentes e violência;

. Índice de ações de promoção de saúde - proporção de unidades que realizam ações voltadas para o autocuidado; promoção da atividade física e alimentação saudável; redução de sequelas e incapacidades; para a redução de danos; e que oferecem informações/cursos de capacitação de cuidadores de idosos;

. Índice de rede de suporte social - proporção de unidades que realizam ações de estímulo à participação das pessoas idosas em grupos da terceira idade, conselhos comunitários, clubes; atividades de identificação e reconhecimento da rede de suporte social para a pessoa idosa; e que informam sobre direitos dosidosos;

. Índice de integração da unidade/serviço com a rede - proporção de unidades com mecanismos estabelecidos de referência e contrarreferência para casos que necessitam de internação; e que possuem integração com equipes do PSF visando à manutenção da capacidade funcional do idoso; 
. Índice de ações de prevenção de distúrbios físicos, cognitivos e de acidentes e violências - proporção de unidades que oferecem uma abordagem pre ventiva com aplicação de instrumentos de avaliação e testes de triagem para detecção de distúrbios cognitivos, visuais, mobilidade, audição, depressão, comprometimento da funcionalidade, distúrbios nutricionais e isolamento social; e que realizam ações de prevenção de violências e de acidentes no domicílio;

- Índice de identificação e notificação dos casos de violência contra idosos - proporção de unidades que realizam ações voltadas para a identificação de casos de idosos vítimas de acidentes e violência; eque notificam sistematicamente os casos de violência contra idosos para autoridade policial ou M inistério Público ou consel hos municipais e estaduais do idoso ou secretarias municipais e estaduais de Saúde ou Secretaria de Desenvolvimento Social;

. Índice de registro e análise dos dados - proporção de unidades que realizam o registro do atendimento aos acidentes/violências classificados de acordo com a CID-10/DSM -IV; e que fazem análise sistemática dos registros.

Indicadores do nível hospitalar geral, emergência e unidade de referência:

. Proporção de unidades com UTI adequada aos requisitos básicos;

. Proporção de hospitais com recursos tecnológicos (mínimos e indispensáveis) adequados e suficientes;

Proporção de unidades que permitem a permanência de acompanhante familiar para idosos internados;

. Proporção de unidades que possuem equipes mínimas para internação/assistência domiciliar;

. Proporção de unidades que oferecem uma abordagem preventiva com aplicação de instrumentos de avaliação e testes de triagem para detecção de distúrbios cognitivos, visuais, mobilidade, audição, depressão, comprometimento da funcionalidade, distúrbios nutricionais e isolamento social;

. Proporção de unidades que recebem ou encaminham pacientes do PSF/Pacs, CAPS, hospitais, outras unidades de saúde, M inistério Público, delegacias, conselhos de direitos, Secretaria de Desenvolvimento social e organizações não governamentais (ONGs):

. Proporção de unidades que realizam al guma ação de prevenção aos acidentes/violência;

- Proporção de unidades cujos profissionais recebem capacitação para identificação e atendimento dos casos de idosos vítimas de acidentes e violência;

Proporção de unidades que já ouviram falar e já leram a PNRMAV, a Política Nacional de Saúde da Pessoa Idosa e o Estatuto do Idoso;

. Índice de recursos humanos para atendimento ao idoso vítima de acidentes e violências em hospital de referência - proporção de hospitais de referência para 0 atendimento às vítimas de acidentes e violên- cias com número mínimo de profissionais indispensáveis para seu funcionamento; e que possuem profissional com formação em gerontologia;

. Índice de identificação e notificação dos casos de violência contra idosos - proporção de unidades que realizam ações voltadas para a identificação de casos de idosos vítimas de acidentes e violência; que notificam sistematicamente casos suspeitos ou confirmados de violência contra os idosos; e que enviam a notificação de casos de violência contra idosos para autoridade policial ou Ministério Público ou conseIhos municipais e estaduais do idoso ou secretarias municipais e estaduais de saúde ou Secretaria de Desenvolvimento Social;

. Índice de registro e análise dos dados - proporção de unidades que possuem pessoal capacitado para registro sistemático da informação; que realizam o registro dos casos de idosos vítimas de acidentes e violência de acordo com a CID-10 ou DSM -IV; e que fazem análise sistemática deles.

Indicadores do nível hospitalar atendimento psiquiátrico:

. Proporção de unidades que possuem profissional com formação em gerontologia;

. Proporção de unidades que possuem equipes mínimas para internação/assistência domiciliar;

. Proporção de unidades que oferecem uma abordagem preventiva com aplicação de instrumentos de avaliação e testes de triagem para detecção de distúrbios cognitivos, visuais, mobilidade, audição, depressão, comprometimento da funcionalidade, distúrbios nutricionais e isolamento social;

- Proporção de unidades que possuem registros sobre os atendimentos às pessoas em situação de violências/acidentes;

- Proporção de unidades que já ouviram falar e já Ieram a PNRM AV, a Política Nacional de Saúde da Pessoa I dosa e o Estatuto do Idoso;

- Índice de identificação e notificação dos casos de violência contra idosos - proporção de unidades que realizam ações voltadas para a identificação de casos de idosos vítimas de acidentes e violência; e que enviam a notificação de casos de violência contra idosos para autoridade policial ou M inistério Público ou conselhos municipais e estaduais do idoso ou secretarias municipais e estaduais de saúde ou Secretaria de Desenvolvimento Social;

. Índice de registro do atendimento pela CID e de pessoal capacitado para esse registro - proporção de unidades que realizam o registro dos casos de idosos vítimas de aci dentes e violência de acordo com a CID10 ou DSM-IV; e que possuem pessoal capacitado para registro sistemático da informação.

Indicadores do nível da reabilitação:

. Proporção de unidades que possuem mecanismos próprios ou integração com a rede de serviços para suporte para laboratório de radiologia e de patologia clínica; 
. Proporção de unidades que possuem protocolos para 0 atendimento de reabilitação aos idosos vítimas de acidentes/violências;

. Proporção de unidades de referência que realizam as principais atividades propostas para seu nível de complexidade;

. Proporção de unidades que realizam atividades voltadas para reinserção na vida comunitária, ações de promoção do autocuidado, ações de fomento da rede de solidariedade para idosos frágeis e sua família e ações de estímulo à participação dosidosos em grupos de terceira idade, consel hos comunitários;

- Proporção de unidades que possuem equipes multidisciplinares para a reabilitação domiciliar dos idosos vítimas de acidentes e violências;

. Proporção de unidades que oferecem uma abordagem preventiva com aplicação de instrumentos de avaliação e testes de triagem para detecção de distúrbios cognitivos, visuais, mobilidade, audição, depressão, comprometimento da funcionalidade, distúrbios nutricionais e isolamento social;

- Proporção de unidades cujos profissionais recebem capacitação para identificação e atendimento dos casos de idosos vítimas de acidentes e violência;

. Índice de recursos humanos de unidades primárias para atendimento ao idoso - proporção de unidades primárias que possuem equipe mínima de profissionais indispensáveis para seu funcionamento; e que possuem profissionais com formação em gerontologia/geriatria;

. Índice de recursos humanos de unidades intermediárias para atendimento ao idoso - proporção de unidades intermediárias que possuem equipe mínima deprofissionais indispensáveis para seu funcionamento; e que possuem profissionais com formação em gerontologia/geriatria;

. Índice de recursos humanos de unidades de referência para atendimento ao idoso - proporção de unidades de referência que possuem equipe mínima deprofissionais indispen sáveis para seu funcionamento; e que possuem profissionais com formação em gerontologia/geriatria;

. Índice de integração da unidade/serviço com a rede - proporção de unidades que possuem integração com equipes do PSF visando à manutenção da capacidade funcional do idoso; e recebem ou encaminham pacientes do PSF/Pacs, CAPS, hospitais ge rais, psiquiátricos, unidades de saúde, M inistério Público, delegacias, conselhos de direitos, Secretaria de Desenvolvimento/Assi stência/Ação Social;

. Índice de registro e análise dos dados - proporção de unidades que realizam o registro do atendimento aos acidentes/violências classificados de acordo com a CID-10; e que fazem análise sistemática deles. 